\title{
Identity, mental health and work: How employees with mental health conditions recount stigma and the pejorative discourse of mental illness
}

\section{Hadar Elraz /Human Relations}

\section{Abstract}

This article asks how identity is constructed for individuals with mental health conditions (MHCs) in the workplace. It takes especial regard to how MHCs are discursively situated, constructed and reconstructed in the workplace. Employees with MHCs face a difficult situation: not only do they need to deal with the stigma and discrimination commonly associated with MHCs, but they must also manage their health condition whilst adhering to organizational demands to demonstrate performance and commitment to work. Discourse analysis derived from 32 interviews with individuals with $\mathrm{MHCs}$ delineates how these individuals feel both stigmatized and empowered by their MHCs. The findings address three discursive strands: (i) a pejorative construction of mental illness in employment and society; (ii) contesting mental illness at work by embracing mental health management skills; and (iii) recounting mental illness through public disclosure and change. This article enhances understanding of how the construction of positive identity in the face of negative attributions associated with MHCs contributes to literature on identity, organizations and stigma as well as raising implications for policy and practice.

Keywords disability, discourse, employment, mental illness, mental health, MHC

\section{Introduction}

Mental health conditions (MHCs) are considered a global challenge (Collins et al., 2011) and one of the leading causes of disability worldwide (NIMH, 2015). Together with the increased awareness of common mental health experiences 1 worldwide (Fryers et al., 2005), mental health and its attendant issues are incredibly important and topical at both a social and political level (Kessler et al., 2005). Yet there is a relative dearth of knowledge about the manifold ways in which MHCs are experienced or enacted within organizations. This is because extant studies often have an impoverished conceptual understanding of mental health and other invisible conditions within the workplace2 (Clair et al., 2005; Santuzzi and Waltz, 2016). Such studies are underpinned by a broad conceptualization of mental health at work, and characteristically focus on the persistence of explicit forms of discrimination and stigma (The Sainsbury Centre for Mental Health, 2007). The stigma associated with MHCs is an emergent concern in the workplace and is now increasingly being challenged by anti-discrimination legislation, as well as disability, mental health promotion and anti-stigma campaigns (Mindful Employer, 2016; Time to Change, 2014).

Although there are debates pertaining to the naming, construction and organization of mental health, the predominant understanding of MHCs is still refracted through historical context, stigma and prejudice (Bracken and Thomas, 2005; Evans-Lacko et al., 2013). Moreover, there is an information deficit regarding both how people with MHCs construct their identities within work settings and what mechanisms they use to maintain their employment (Leufstadius et al., 2009; Santuzzi and Waltz, 2016). This article departs from the aforementioned broad and disempowered conceptualizations of MHCs. It does so by problematizing the assumption that MHCs necessarily correspond with identities that are marginalized or victimized within employment and society (Scull, 1979; Tsang et al., 2007). Further, the article does not aim to directly examine experiences of work/non-work discrimination (CIPD, 2006; Equality Act, 2010). Rather, the objective here is to explore how individuals with MHCs operate within and retain employment and how they challenge and resist hegemonic understandings of mental health at work through their own experiences. This article mobilizes a qualitative and 
discursive research approach to analyse a large dataset emerging out of interviews with individuals with MHCs. The methodology and analysis is grounded in a critical poststructuralist tradition, which aims to understand the tensions and underlying mechanisms that are embedded in employment and society at large in this particular context (Foucault, 1982; Knights and Willmott, 1999). From this perspective, one would examine the issue of mental health at work in terms of an arena in which identities and beliefs are created, contested and recreated (Alvesson et al., 2008). As there is not a well-developed body of research critically exploring identities and MHCs at work, the analysis presented here provides important insight into the prominent approach for studying mental health in the workplace (Corrigan and Matthews, 2003; Thoits, 2016). By demonstrating the complex nexus of identity meanings in this context, in a way that moves beyond current doxa about people with MHCs, this article helps in shifting the debate from emphasizing the negative consequences of mental health stigma and pejorative attributions (The Lawyer, 2009; Tse, 2004).

The emphasis instead concerns how assumptions that have come to be taken for granted are actively being transformed for the better by foregrounding the experiences of people with MHCs who attempt to maintain employment and earn socioeconomic legitimacy through work. This perspective subsequently informs theory, attitudes and practice. Given that mental health is a broad category spanning from numerous common mental health experiences to more severe and long-term MHCs, the approach used here offers wider implications beyond MHCs. That is to say, although this article explores how identities are negotiated and resisted within the specific context of MHCs, its implications can be expedient for understanding the broader meanings associated with employment and mental health. The article is structured as follows. First, I examine literature on identities in the workplace from a critical and poststructuralist perspective, as well as literature on mental health and marginalized identities generally. Second, I situate the theoretical and methodological context for the research, before proceeding to discuss the methods, and delineate the key findings emerging from the analysis and the implications of the study for theory and practice.

\section{Critical perspective on identities at work}

The notion that identities are bounded within discourse assumes that identities are produced through the operation of knowledge/power relations. This Foucauldian approach to studying identities at work has been particularly influential within the study of organizations, especially in research that adopts a critical perspective (Alvesson et al., 2008). Influenced by poststructuralist theorizing on discourse and identity, these studies conceptualize identity as constituted through power dynamics, as well as being fluid, context-bound and compounded by a number of identity meanings, namely, subject positions (Dean, 1999; Foucault, 1977). Hence, an individual's identity construction emerges out of manifold subject positions (Musson and Duberley, 2007), each of which constitutes a part - without forming a totality - of identity (Holmer-Nadesan, 1996).

Such studies examine how cultural, political and historical practices are reflected in identity formation, and inform the dynamic relationship between organizational discursive regulation and employees' identity constructions. Discourse and the subject positions emerge, in turn providing meaningful ways of being from which individuals come to identify with and gain an understanding of themselves. This stance on identity can thus be understood as a site for the production of knowledge (Hardy and Thomas, 2013) that enables us to explore contemporary discourses and practices of contestation. This conceptualizing is therefore vital for understanding the construction of MHCs, as well as how individuals with MHCs understand themselves and live their lives. In so doing, this approach enables us to study how and why individuals respond in different ways to mental health and work discourses. Studying identities critically, in terms of how they are a site for knowledge production, is important 
for gaining insight into both individual experiences and a broader range of social and employment concerns relevant to a broad demographic of workers (Collinson, 2003; Thomas, 2009).

Paradoxically, although identity within organizations has been explored critically in a variety of respects (Ainsworth and Hardy, 2009; Garnsey and Rees, 1996; Riach, 2007; Rumens and Kerfoot, 2009; Van Laer and Janssens, 2011), scant consideration has been shown to the identities of people with disabilities or long-term health conditions (Foster and Wass, 2013), especially less visible conditions like MHCs (Clair et al., 2005). The social construction of mental health conditions Although MHCs have occupied industrial psychologists since the First World War (Rose, 1999), and whilst both common mental health experiences and MHCs worldwide are due to be even more costly in the future (Collins et al., 2011), these issues still remain under researched, particularly within the setting of identities and the contemporary workplace.

At a societal level, various studies demonstrate how mental health categorization and resistance can have profound implications for identity. For example, Anspach (1979) shows how societal activism on behalf of former mental health patients attempted to create a positive political identity for mental health. More recently, Thoits (2016) put forward the notion of identity deflection, which is a resistant practice towards mental health stigma that people with MHCs can perform in order to refuse adoption of pejorative identity attributions (Thoits, 2016). More importantly yet still, studies have also demonstrated how positive engagement with some mental health symptoms for bipolar disorder can engender positive identity attributions for people with MHCs (Forgeard, 2016). In terms of health management, studies related to mental health treatment demonstrated how, although mental health services are no longer provided in the form of asylums, they can still benefit from empowering individuals with MHCs. Hyde and Davies (2004), for example, express the dual consequence that contemporary cultural mentality which reinforces that of the asylums may lead to limited possibilities for the co-production of care between caregivers or for patients themselves to lead the management of their own MHCs (Hyde and Davies, 2004). In a similar vein, Randall and Munro (2010) capture the scepticism of mental health professionals towards the acceptable normalizing techniques of treating mental health patients.

Drawing upon interviews with mental health practitioners in the voluntary and statutory sectors in Scotland, the authors illustrate how instead of popular and normalizing techniques for dealing with MHCs appearing through modalities such as medical diagnosis and medication, the practitioners suggested alternative forms of care provision and treatment focused around patients' own experience and skills of self-management, and led primarily by them. As far as the workplace is concerned, quantitative studies document the billions of working days lost every year because of mental health related absence (The Sainsbury Centre for Mental Health, 2007) when psychosocial constraints (e.g. stress or other temporary or transient pressures) have a significant effect on employees' mental health (Trudel et al., 2009). For example, when people are working under high levels of psychological strain, they are more likely to have higher occurrences of depression (Mausner- Dorsch and Eaton, 2000). Similarly, tenseness, stress, and other unsafe or threatening working conditions are designated as contributing factors to work-related mental illness (Goldman and Lewis, 2008). Interestingly, mental-health-related absence from work is not only associated with low workplace performance, but also with pejorative attributions, oppression, social rejection, isolation, discrimination, unemployment and low income (Tsang et al., 2007).

Resultantly, the area of mental health and work represents an interface in which the nature of the illness and the social stigma that surrounds it can result in particular manifestation of identities at work. For employees with MHCs, this is specifically problematic because they may not only be 
impaired by the constraints of the health condition itself, but, rather, also by other workplace pressures as well as stigma, negative stereotypes and common myths ('it is not healthy for people with MHCs to work') (Krupa et al., 2009).

The impact of such widespread stigma and discrimination can be detrimental for the ability of people with MHCs to cope with work, perform, be able to communicate or socialize. All these can, in turn, negatively affect the employment opportunities of people with MHCs as well as their having to endure feelings of lack of competence at work (Tsang et al., 2007). Negative attributions and pernicious prejudice have also been shown to contribute to other problematic issues, such as limited selfdisclosure to work colleagues and reduced managerial awareness of MHCs (Martin et al., 2015). This is because, although people with MHCs are encouraged from an occupational health perspective to disclose their condition to employers (Hatchard, 2008), they often fear that the stigma of mental illness may jeopardize their future career prospects (Occupational Health, 2006).

This is problematic because limited self-disclosure, in fact, may have other detrimental effects for these individuals, ranging from limited access to appropriate treatment and diminished workplace performance, to personal anxiety and preoccupation with potential discrimination (Honey, 2003), selfstigma (Corrigan and Watson, 2002), and a sense of not being able to fulfil one's full work potential (Gelb and Corrigan, 2008). Consequently, many individuals with MHCs are trapped in a cycle of unemployment and low-status jobs, and are marginalized from work integration (Marmot Review, 2010). Although research demonstrates how beneficial work can be for individuals with MHCs, providing a sense of belongingness and meaning, as well as occupational and health rehabilitation (Leufstadius et al., 2009), the above workplace-related constraints experienced by these individuals may restrict their ability to gain, maintain or thrive in work.

This problem is exacerbated, of course, within tight labour markets that characterize periods of recession - as was the case when these data were generated - when employers are more demanding and selective. These tight labour markets intensify pre-existent pressures to enhance people's career prospects by working harder, maximizing their performance, and strengthening their commitment to their employer (Costea et al., 2012). In light of these constraining employment situations, the struggle of those with MHCs may be somewhat unsurprising. What is significant, however, is that, although these studies provide a good understanding of how individuals with MHCs relate to the pejorative meanings associated with MHCs, there is a relative dearth of literature demonstrating how these individuals refute the predominant discourses in employment and society, and the subsequent impact this has on their lives and employment situation. For this reason alone, studying identities within this context is crucial.

\section{Methods}

The methodology is grounded in the critical tradition within organizations and a discursive approach to analysing interview texts (Bergström and Knights, 2006; Holmer-Nadesan, 1996). The data were generated as part of a large UK-based project sponsored by the ESRC during 2010-2013. The primary method for data generation involved 60, one-hour interviews that I conducted, recorded and transcribed. This included interviews with individuals with MHCs, health professionals, HR professionals and line managers, employees in third and public sectors, and mental health organizations, as well as senior staff in the private sector. The sample is heterogeneous in terms of gender, the range of MHCs covered (mood disorders, anxiety disorders, psychosis disorders), level of severity, age and occupation. It is worth noting my personal and professional motivations for conducting this research. On a personal level, I have relatives and friends with MHCs, whilst I 
personally have undergone transient common mental health experiences such as stress and anxiety in both work and non-work settings.

On a professional level, my engagement as an employment specialist for individuals with health conditions and disabilities made me acutely aware of the profound struggle involved in having a $\mathrm{MHC}$ within a workplace. What struck me most is how individuals with MHCs tend to suffer in silence. Not only do they have to cope with a challenging and enduring condition with a variety of debilitating symptoms, but they also must do this whilst receiving little recognition from society. Late diagnosis, the often complex manifestation of MHCs, and the subsequent detrimental impact this has on their lives encouraged me to examine their experiences within organizations, in the hope that this would enhance understanding and, in turn, improve the employment situation of others (Elraz, 2016). Using a snowball sample (Browne, 2005), the information about the study was disseminated via a leading mental health charity in the UK, as well as interrelated mental health support groups and organizations. Individuals with MHCs who were willing to participate in the study were interviewed.

Ethical guidelines were followed throughout the various stages of the research, including anonymity and confidentiality, as well as a rough interview guide which enabled the participants to discuss only the issues they found important or felt comfortable discussing (Ghauri and Grønhaug, 2002). To inductively identify relevant topics to include in the interview guide, I conducted a number of pilot interviews with both mental health professionals and individuals with MHCs. Issues over managing the MHC within the workplace setting and the social attributions towards MHCs were the predominant emergent themes. Following on from this, 32 repeat interviews were conducted with 16 working individuals who had one of the following MHCs: bipolar, depression, obsessive compulsive disorder and schizophrenia.5 As the preliminary analysis of these data demonstrated the dynamic nature of identities as they pertain to mental health and work, it thus became clear that exploring these issues over time and on an ongoing basis would be expedient for researching this interface (see also Santuzzi and Waltz, 2016). Each individual was therefore interviewed twice over a period of 18 months to examine identity dynamics in relation to changing circumstances. The interview guide was comprised of the following questions: Could you talk about your health condition in relation to your employment? Could you tell me about your day-to-day experiences inside and outside of work? What are your work experiences in relation to mental health? How do you get on in the workplace? How do you think others in this organization (colleagues/manager) view you or your work performance?

The process of data analysis aimed to identify emergent themes in the data, alongside classifying the discursive practices through which people with MHCs engage with hegemonic discourses on mental health and employment as part of their everyday identity construction. The issue of relating to one's mental health identity emerged both spontaneously and in response to questions about mental health and work. That is to say, in their attempt to construct positive workplace identities, the participants positioned themselves in relation to how they perceived the notion of mental health at work. Although issues of identity construction were visible in the analysis of the entire dataset, they came out most strikingly from the repeat interviews. As such, whereas the entire dataset was used to gain a better understanding of mental health at work and the conceptualization of identities, the analysis below predominantly draws on findings from the repeat interviews as a way through which to maximize our understanding of identities in this context and to contribute to theory development (Santuzzi and Waltz, 2016). The analysis of the entire dataset was undertaken in three stages, although these remained relatively fluid and shifted iteratively between the different levels of analysis. The first stage involved identifying explicit themes within the text. Subsequently, notions of mental health and identity attributions (e.g. 'people think about mental health', 'my health condition', 'mental illness' 
and so on) were identified to examine the interrelationship between the construction of various discursive resources and themes explored in the study.

Of especial interest was how the mental health identity itself was used to promote or dismiss particular ideas adopted within the interview texts. The final stage of the analysis involved a finegrained reading of the data so as to identify nuances and link these back to theory and the research aims (Alvesson, 2003). The main findings emerging from the analysis concerned how people with MHCs continue to feel oppressed whilst simultaneously feeling empowered by their mental health subject position. Three main themes were identified: (i) a pejorative construction of mental illness in employment and society; (ii) contesting mental illness at work by embracing mental health management skills; and (iii) recounting mental illness through public disclosure and change. Whilst the first theme demonstrates how individuals with MHCs cope and situate themselves within the boundaries of the pejorative mental health discourse, the other themes show an attempt to redefine and introduce new meanings that can offer a better understanding of mental health at work. Finally, vignettes were selected that best illustrated the main themes in the findings. The first theme, presented below, discusses a disempowered discursive stance, which affirms the existing stigma and discrimination towards MHCs in the workplace.

\section{A pejorative construction of mental illness in employment and society}

The participants' comments portray how negative societal representations of MHCs translate into the workplace. Of particular note was the fact that the interviewees argued that there is an assumption that people with MHCs are unable to work. The findings demonstrate that there is a putative caring approach in operation here, whereby the weak, delicate and ill person should have work provided for them, but only work that is undemanding, safe and low-status. In other words, a person with a MHC lacks the mental resilience to be a fully functioning employee. Tony, who worked for 20 years as a telecom engineer before taking a part-time support job in a local hospital, illustrates the detrimental effect of becoming unwell on an identity:

Very often a person considers to be what they are by the work they do ... And if you become unwell you lose all that. And then you become possibly 'oh he had a mental breakdown' or 'he is very nervous' or 'he is very anxious' so yeah, it can affect people.

The assumption being made here is that having a MHC precludes someone from being able to work or be fully engaged in the workplace, that is, that the MHC somehow deems the individual unproductive or incompetent in the workplace. Moreover, the disassociation of the person with the $\mathrm{MHC}$ from the notion of being a productive worker strongly influences attitudes within the workplace towards colleagues with MHCs. The interviews suggest that there is a more sceptical association, whereby the invisible nature of MHCs leads to accusations that the illness is not genuine, and, hence, that those declaring a MHC may be simply workshy or 'unable to take the pressure'. Here, again, the assumption is that someone with a MHC lacks the mental drive to effectively participate in employment.

Brian, a diversity educationalist, observes: 'Somebody thinks someone has a mental health condition - the only thing they think he is capable of is - he can clean the floor; he can stack shelves'. Similarly, Bret, a radio engineer, recounts: 'I don't think people associate mental illness with people who are functioning in high-status jobs ... People associate mental illness with people who can't work'. With regard to these specific extracts, what is being suggested is that the societal view about mental illness operates as a 'blanket discourse' that overrides and colonizes other work-oriented subject positions, which is to say that the person is deemed to be, first and foremost, 'mentally ill'. Consequently, the 
person with the MHC is portrayed within the workplace as someone who is incapable of adequately performing within the demanding parameters of skilled work or of living up to neoliberal workplace expectations of optimal performance (Munro, 2012).

However, what is also significant from the analysis is the way in which this pejorative notion of mental

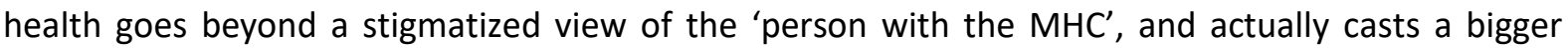
shadow over common mental health experiences, such as transient stress or anxiety at work. Patrick, a university lecturer, states: I have had conversations with people as if: 'Oh I don't want people to know I am off on stress it makes me feel as if I am weak, you know' ... a lot of institutions maybe the more macho institutions that people have to work in are more like that, I think. The attribution of a reduced capacity to perform is, as with other MHCs, associated with absence owing to stress or with other symptoms of suffering with stress at work, such as an inability to cope with work, handle the workload, or a failure to possess the requisite organizational skills for a managerial position. This is seen as directly conflicting with the expectation that employees are committed and able to perform to a prescribed standard. Whilst these findings reinforce the societal view in which people with MHCs are looked at pejoratively, there is a marked emphasis on how this construction manifests in the workplace. Below are two alternative discursive strands emergent in the interviewees' texts. In contradistinction to the pejorative construction, these strands demonstrate how the speakers embrace the experiences of living with a MHC and actively seek to share these experiences with others.

\section{Contesting mental illness at work by embracing mental health management skills}

The respondents highlighted a number of positive meanings associated with embracing the experiences of living with a MHC. In sharp opposition to the view that the person with a MHC is incapable of holding down work, this discursive strand in the speakers' talk demonstrates how the participants utilize their skills and experiences as an advantage in the workplace. In their talk, the respondents articulated how by gaining special skills as a consequence of their MHC, they are better positioned in their employment situation compared to other colleagues. For instance, some respondents argued that the challenges of living with a MHC meant that they had developed unique skills and insights, which were of benefit to them in life and in work. For example, Jo, a social worker, suggests that people with MHCs are especially resilient at work, as they can effectively manage their $\mathrm{MHC}$ and deal with the demands of the job while coping with stress at work:

Stress sometimes seems a weakness, that people should manage themselves better. And I am probably guilty in that point of view, because I think 'well if I manage it everybody else should manage it' ... It's about having the responsibility ... so I tend to take a couple of days off a month mainly for study but so that I am not doing weeks and weeks and weeks without a break.

The embracing of $\mathrm{MHC}$ as a positive quality in both work and non-work environments is reflected in the interviewees' texts. In addition to embracing $\mathrm{MHC}$ as a positive quality, it is advised that participants' mental health management skills could be formalized within the organizational culture more generally. The participants address more positive qualities by expressing their excessive dedication to the workplace even in times of unwellness. Martin, who holds a managerial role in a bank, comments: 'I probably wasn't well all the time but I still didn't have any days off sick'. Melanie, a university lecturer, likewise observes: 'My line manager happens to be very open minded and very open to work with me and I think they see that a lot of people with disabilities actually work harder than other people'. Chris, an IT consultant, commented: 'The people I worked with really liked me because I used to work so hard'. Hard work, demonstrating commitment and selfless dedication, is 
seen as a necessary aspect for self-managing and performing at work. Interestingly, these positive qualities are positioned in the speakers' texts, in contradistinction to the view that people with marginalized identities do not meet workplace expectations of unrestrained performance (Acker, 1990; Munro, 2012), thus providing an opposing narrative to the disempowered, underperforming subject position identified in the first discursive strand.

What is also articulated by the participants here is how the embracement of a MHC ties in with broader meanings associated with the 'business case for disability' (Zanoni and Janssens, 2004). Here, the participants argue that the qualities and experiences acquired through having a $\mathrm{MHC}$ can benefit the workplace (Jammaers et al., 2016). Furthermore, the participants' extracts demonstrate how medical insight into their condition is yet another aspect that helps them gain positive meanings from their identity construction. Chris comments:

I have been doing that for years. I self-manage myself by taking mood stabilisers, antidepressants. I have got a cocktail of those at the moment so it's finding one that works to get you up to a level where you can function. The emphasis here is on taking control, selfmedicating, and deciding when and how best to do this.

This example, in and of itself, shows the application of skills and expertise to come to know the condition, as well as an identification with the need to manage it. In conjunction with this, at the same time that the participants frame their specialist knowledge of managing MHCs as a competitive advantage at work, they also further address the coping skills required to maintain employment and remain healthy at work. Fred, a paint technician, argues:

I am a pretty strong character, you know. I am a tough cookie. So I can be weak, tearful but that's part of the condition, but I am a strong character. I think sometimes, I don't think people realise how strong a character you are, because they don't have any reference, because they never suffered from it themselves.

The emphasis here on having unique skills and life experiences, not to mention possessing inordinate degrees of mental toughness, with direct applicability to the workplace, is in stark contrast to the pejorative notion of 'mental illness' delineated above. Viewing people with MHCs as being different in a positive sense signals a move away from viewing one's 'difference' as stigmatized or pejorative, towards viewing it as a competitive 'advantage' that can benefit the employer and empower the individual, thus culminating in a positive identity construction. Furthermore, such attempts to recalibrate mental health identity as a unique advantage also represent an attempt to destabilize predominant workplace discourses, by not simply normalizing the identity, but rather embracing it as a unique difference. However, some employment contexts appear to be more constraining than others, heightening the need for mental health management whilst, simultaneously, restricting the opportunities to do so. These instances are important because they foreground the question of whether or not to declare a MHC, as well as highlighting the importance of specific workplace contexts in terms of shifting the negative attributions associated with MHCs.

\section{Recounting mental illness through public disclosure and change}

Public disclosure is an act that both challenges and recounts the stigma and secrecy often associated with having a MHC. Patrick argues: 'A lot of people I talk to, say: "oh I think my wife's got that", or "my friend's got that" or "I think I have got that as well", when you have a little chat'. Melanie comments:

... for everybody who does speak out, it will help other people ... quite often a lot of people tell me about their own conditions or about their own experience of it because they'll know 
that I'll understand it ... so people tell me of their own experiences of breakdown or mental health problems.

As seen above, public disclosure can thus be viewed as an act of generosity, which encourages others to discuss and be open about their mental health experiences. Those who declare, in turn, see themselves as champions and pioneers. By publicly disclosing in a range of localized settings, such as self-help groups or other contexts with friends and acquaintances, the participants identify with the MHC as a legitimate health condition, thus recounting the aforementioned mental health stigma. Bret argues:

I am a very open person, you know, I don't force things down people's throats but if somebody says, you know: 'What are you?' I just say, you know, 'it's bi-polar, manic depression', whatever you want to call it. I have got no problem with being, you know, having a badge on me [laughter]. I think it's part of me. Why should I hide away? I am not a freak you know; I have got a condition. And then if I see other people and then I think if I gave them a bit of insight and knowledge, maybe that'd save them from going through some of the things.

By publicly disclosing their MHCs, the participants resist the predominant meanings that are associated with mental health and employment. Instead, their texts testify to a concerted attempt to legitimize the identity associated with a MHC. One can see an example of this position in Bret's extract above, where he takes pride in the experience of living with the condition and of sharing that selfknowledge with others. Parallel to this, a number of the participants have taken disclosure into more public settings, in order to open up discussion around mental health, and so as to encourage other employees to talk about their own experiences. For example, Cliff, a lawyer and founder of a mental health charity, uses his own skills and mental health experiences as a platform to promote mental health at work. He argues:

Having high profile role models within the organisation is a good way of establishing cover for more junior stuff so that they can see there is a commitment and it doesn't affect your career in that way ... people who want to 'come out' within the organisation. To provide a personal account of how the individual is being supported and able to continue working, in their own experience.

With reference to the extract above, we can see how speakers use their position within the workplace to engender change in a broader organizational context. The accommodating responses received with managing the MHC following the disclosure may therefore lead other colleagues to share their own experiences of illness. Interestingly, the findings demonstrate how, by taking a micro-political role with respect to their MHCs, the participants act as advocates who promote openness towards mental health within their workplace, whilst simultaneously stressing the benefits that open discussion about mental health would have for businesses generally. Bruce, a partner in an international accountancy corporation, states that his personal experience of a MHC motivated him to encourage other employees to come forward and to develop managerial strategies that would directly lead to change within the organization. Like Cliff, Bruce also stressed how increasing awareness around mental health would be helpful, not only for the individual but also for the organization's overall performance:

If it happened to me then I suspect it happens to an awful lot more people. And understanding that, kind of, one in four people some time in their life will have it [mental illness] ... Because I have been treated very well on my return, you know, I was very engaged with the firm. I won lots of work, and I have done that mainly because I feel so good about the place. So that led me to think about what I could do to make it even better here, and potentially also, with other 
sorts of organisations, to get that awareness culture ... If we could get more onto the front foot around preventative, awareness-raising issues.

Inaugurating forms of change at an organizational level that would promote mental health awareness and intentional disclosure recounts MHCs mode of subject position, and opens up the possibility for alternative and positive meanings in this discursive construction.

\section{Discussion}

This article set out to explore the experiences of individuals with MHCs in the workplace, reflecting on how broader meanings associated with mental health at work are discursively situated, constructed and reconstructed. The main findings presented above show the manifold ways in which people with MHCs continue to both feel oppressed and empowered by their mental health subject position. Three interrelated themes were identified in the analysis: (i) a reaffirmation of the social stigma and pejorative construction of a mental health subject position within work; (ii) a recasting of the mental health subject position as a less productive mode of subjectivity through transferring mental health management skills to the employment context; and (iii) a reconstruction of mental health as a disempowered mode of subjectivity via public disclosure and change. These three findings each constitute a valuable contribution towards understanding how the notion of mental health at work emerges and is (re)constructed within the workplace.

By articulating how individuals with MHCs construct positive identities in the face of negative attributions, this article makes crucial interrelated contributions to policy-making and to organizational literature, both with respect to critical identity studies and, more specifically, literature on stigmatized identities at work. Each of these will now be dealt with in turn. The first contribution made by this article relates to the field of identities at work. By delineating how individuals with MHCs construct and negotiate identities at the intersection of multiple constraints of discursive regulation, this critical identity approach problematizes current doxa on power and mental health by showing the power relations between individuals' own understanding of the self, discourse and subject positions (e.g. Alvesson and Willmott, 2002).

The analysis, ultimately, indicates that the process of constructing identities involves both discursive meanings and a set of self-management practices, such as the skills and forms of self-knowledge required for managing $\mathrm{MHCs}$, which, in turn, provides the individual with self-understanding about how they relate to their identities. In so doing, this research makes a contribution to theory development within critical literature on identities, and casts light on an underexplored area related to identities, organizations and self-management (Starkey and Hatchuel, 2002). One key insight is that the empowered position gained through self-management can not only assist in critically theorizing identities (Randall and Munro, 2010), but it also bears significant micropolitical implications in terms of shifting pejorative meanings in the context of identities and marginalization, which has considerable implications for individuals' working experiences. In accordance with other studies that have demonstrated the fluid, insecure, unstable, fragile, and even anxious (Knights and Clarke, 2014) or contradictory nature of identity construction (Collinson, 2003), this study similarly emphasizes the multiplicities and nuances of the strategies through which individuals procure a greater understanding of their identity.

The theoretical framing of discourse and identities provides a sophisticated understanding of the mechanisms employed within mental health discourses and subject positions, specifically within the context of resistance and compliance towards discursive regulation (Kondo, 1990; Thomas and Davies, 2005). The article thus contributes to developing theory as it demonstrates how individuals negotiate 
their own identity vis-a-vis this process to engender a more empowered identity with the setting of MHCs. The article has also demonstrated how the context-bound and situated nature of identities is more challenging to articulate within the specific settings explored here, thus calling into question the nature of identity construction for other individuals with MHCs at work. In contradistinction to both extant critical literature (e.g. Hyde and Davies, 2004; Randall and Munro, 2010) and less critical studies in this field (e.g. Thoits, 2016), the analysis presented here specifically focuses on identities and individual experiences within work, therefore providing expedient insight into this crucial interface. This latter point connects directly with the second contribution of this article, which concerns the critical exploration of marginalized identities at work (e.g. Slay and Smith, 2011), particularly as it pertains to stigma, disability and health conditions. Studies have ably demonstrated how individuals with disabilities and health conditions face challenges in resolving the tension between organizational discourses that propagate an excessive performance culture unfettered by non-work-related issues (e.g. Denissen, 2010).

By documenting how individuals with such identities position themselves in relation to organizational discourses concerning high performance, this analysis extends previous research that explores how 'historically subordinated social groups' (Jammaers et al., 2016: 1380) negotiate the complex dynamic of resistance, contestation and identity regulation in the workplace. Whilst the interviewees related their MHCs to the business case for disability and the organizations' expectations for workplace performance (Acker, 1990; Gelb and Corrigan, 2008; Omansky-Gordon and Rosenblum, 2001; Zanoni and Janssens, 2004), they also reversed the disempowered subject position seen in the first strand of the findings. By introducing additional meanings that went beyond a straightforward performative repositioning and pointing to ways of altering the association with performance as 'doing more' (Costea et al., 2012), we can see alternative discursive meanings that, in turn, challenge current understanding of workplace performance (Jammaers et al., 2016).

These alternative discursive meanings therefore provide insights that go beyond the business case/performative positioning towards alternative meanings in relation to acting upon the stigmatized subject position or even addressing 'difference in its own right' (e.g. Overboe, 1999; Roberts, 2005). This, in turn, opens up new avenues for cultural openness towards mental health at work. These insights also provide a more nuanced understanding of the field of stigma and workplace disclosure. Although the analysis does not propose a definitive pattern of when disclosing or concealing is more possible, as it pertains to specific sectors/jobs or organizational and individual contexts per se (Clair et al., 2005), it goes further than what is currently known from the literature, particularly by providing a better understanding of the struggle with identifying with a complex and problematic subject position, such as in the case of MHCs.

The analysis suggests how identifying with a MHC places the individual in an incredibly vulnerable situation - one with excessive sets of concerns over disclosing MHCs at work. As there are workplace cultures and practices that are reticent about recognizing MHCs as a debilitating condition, even when the MHC is officially designated as a disability6 (Equality Act, 2010; Santuzzi and Waltz, 2016), the identification with the mental health subject position becomes even more problematic. This article demonstrates how such a process can be related to questioning the individual's ability to interact at work, manage other people or adopt specific roles. Further still, although the literature shows the different motivations for disclosure (e.g. Ely and Thomas, 2001; Friskopp and Silverstein, 1995), this exploration draws attention to the linkages between identification and disclosure.

This was particularly notable with respect to how individuals with MHCs reflect upon their day-to-day experiences and unique set of skills in managing their MHCs (as seen in the second strand), and in 
terms of learning about their disclosure experiences and attempts to engender change in understanding and relating to mental health at work (as seen in the third strand). The analysis demonstrated how the struggle to endure by either disclosing or concealing a stigmatized subject position is related to the individual attempt to be understood (Matthews and Harrington, 2000), build close relationships at work (Greene, 2000), bring change (Creed and Scully, 2000; Taylor and Raeburn, 1995), educate, raise awareness (Bernstein, 1997) or reduce stigma (Kitchener and Jorm, 2004, 2008).

By providing a better understanding on how identification within the context of mental health is manifest in the workplace we can therefore see various ways to relate to the mental health subject position. The analysis therefore casts light upon a problematic issue - one yet to be adequately explored in legal and organizational debates - whereby individuals themselves reposition the discussion around identities, stigma and MHCs in relation to their own health management as well as in relation to hegemonic organizational discourses concerning performance. These acts of personal authenticity that were notable in the findings are therefore deemed fundamentally crucial, not only as they 'render one's everyday existence meaningful' (Rose, 1999: 272), but also as they support individuals in obtaining the workplace recognition and legitimated identities for which they are endeavouring (Foster, 2007; Jammaers et al., 2016).

The critical perspective used in this study provides crucial insights that go above and beyond MHCs, instead allowing for a greater understanding of mental health more broadly. Of course, studying extremes is already established as a useful method for exploring and theorizing across the sciences (Baron-Cohen, 2003; Eisenhardt, 1989; Goffman, 1968). The critical discursive approach underpinning this study afforded insights into broader discourses about mental health and employment through studying MHCs, as these extremes can illuminate broader issues experienced by a far larger population of workers. Indeed, the participants themselves argue that their own knowledge and insight into MHCs provided them with a rare understanding into managing and relating to common mental health experiences shared by others. This complements existing work that has also provided a deeper understanding of transforming mental health stigmatization, which, in turn, can inform 'theory and evidence based interventions that can successfully reduce stigma in all its manifestations' (Bos et al., 2013: 17; see also Holley et al., 2012). While this study offers several interrelated contributions for organizational literature, future research could further explore identities within this setting to assist in both theory development and the creation of better policy in this area. Although the benefits of mental health awareness for both organizations and decreasing mental health stigmatization have been addressed by a host of scholars (Dimoff et al., 2016; Pinfold et al., 2005), recent reviews still show how, despite its prevalence and socio-political relevance, both MHCs as well as common mental health experiences are still underexplored within organizational and managerial contexts, with studies demonstrating how managers, in particular, are lacking the requisite knowledge/training in people management in this area (Martin et al., 2015).

The findings presented here lend support to a pedagogic agenda to advance knowledge by encouraging both policy-makers and HR practitioners to embrace an open culture towards both common mental health experiences and MHCs. That said, future studies could assist in advancing the knowledge base in this area further yet still by exploring how identities are constructed for different $\mathrm{MHCs}$, or in relation to common mental health experiences at work. Future research could also benefit from exploring identities across various sectors (see also Janssens and Zanoni, 2005) and occupations to delineate a more nuanced understanding of identities, as well as the subsequent implications for workers generally. Although the present article could not account for all of these aforesaid issues, and, indeed, may be limited as it concerns how a number of MHCs impact upon the participants' experiences and identities, I would argue that it nevertheless extends our understanding of identities 
in relation to some of the most common MHCs in the workplace. As such, not only does it provide for a better understanding of the social world, but also suggests ways of opening up possibilities for transforming workplace culture in such a way that would, ultimately, benefit other workers.

\section{Acknowledgements}

I would like to thank those who have supported this study, especially the study participants for speaking out about their experiences. I am also grateful for the feedback from the editor Nick Turner and the three anonymous reviewers on previous drafts of the article.

\section{Funding}

This study was supported by funding from Research Councils UK Economic and Social Research Council (grant number: ES/1902023/1).

\section{Notes}

1 Research shows that mental health problems in the community typically refer to the numerous and less severe or enduring common mental disorders, such as depression and anxiety (Fryers et al., 2005; Keyes, 2005). Consequently, I define these as 'common mental health experiences'.

2 I draw a distinction between common mental health experiences and mental health conditions (MHCs) in order to differentiate, firstly, between transient or common experiences of common mental health experiences such as depression and anxiety (Fryers et al., 2005), and, secondly, enduring diagnosable and long-term MHCs (also known as mental illness) that last for 12 months or longer. Whilst all the participants' accounts that are mobilized in this article include diagnosable long-term MHCs (please see Methods section for more information), these conditions share some overlapping markers and symptoms with common mental health experiences, in so far as they relate to the management of emotions, thoughts and behaviours that the study participants draw on in their interview quotes.

3 Such discrimination takes place on an interpersonal level (Lloyd, 2010) and at wider constitutional levels in terms of inequalities in rights and responsibilities, such as reduced citizenship and poverty (Marmot Review, 2010).

4 For further information of the various conditions, see:
http://www.rcpsych.ac.uk/healthadvice/problemsdisorders.aspx (accessed 24 July 2017).

5 As note 4.

6 If the mental health conditions last for 12 months or longer and impact on a person's day-today functioning.

\section{References}

Acker J (1990) Hierarchies, jobs, bodies: A theory of gendered organizations. Gender and Society 4: 139-158.

Ainsworth S and Hardy C (2009) Mind over body: Physical and psychotherapeutic discourses and the regulation of the older worker. Human Relations 62(8): 1199-1229.

Alvesson M (2003) Beyond neopositivists, romantics, and localists: A reflexive approach to interviews in organizational research. Academy of Management Review 28(1): 13-33.

Alvesson $\mathrm{M}$ and Willmott $\mathrm{H}$ (2002) Identity regulation as organizational control: Producing the appropriate individual. Journal of Management Studies 39(5): 619-644.

Alvesson M, Ashcraft KL and Thomas R (2008) Identity matters: Reflections on the construction of identity scholarship in organization studies. Organization 15(1): 5-28. 
Anspach RR (1979) From stigma to identity politics: Political activism among the physically disabled and former mental patients. Social Science \& Medicine Part A: Medical Psychology \& Medical Sociology 13: 765-773.

Baron-Cohen S (2003) The Essential Difference: Men, Women and the Extreme Male Brain. New York: Penguin Press Science.

Bergström O and Knights D (2006) Organizational discourse and subjectivity: Subjectification during processes of recruitment. Human Relations 59(3): 351-357.

Bernstein M (1997) Celebration and suppression: The strategic uses of identity by the lesbian and gay movement. American Journal of Sociology 103(3): 531-565.

Bos AER, Pryor JB, Reeder GD and Stutterheim SE (2013) Stigma: Advances in theory and research. Basic and Applied Social Psychology 35(1): 1-9.

Bracken P and Thomas P (2005) Postpsychiatry: Mental Health in a Postmodern World. Oxford: OUP.

Browne K (2005) Snowball sampling: Using social networks to research non-heterosexual women. International Journal of Social Research Methodology 8(1): 47-60.

Clair JA, Beatty JE and Maclean TL (2005) Out of sight but not out of mind: Managing invisible social identities in the workplace. Academy of Management Review 30(1): 78-95.

CIPD (2006) Firms discriminate on mental illness. Available at: http://www.cipd.co.uk/ pm/peoplemanagement/b/weblog/archive/2013/01/29/firmsdiscriminateonmentalillness-2006-

06.aspx (accessed 24 July 2017).

Collins PY, Patel V, Joestl SS, et al. (2011) Grand challenges in global mental health. Nature 475:27-30.

Collinson D (2003) Identities and insecurities: Selves at work. Organization 10(3): 527-547.

Corrigan P and Matthews AK (2003) Dealing with the stigma of mental illness by coming out of the closet. Journal of Mental Health Services 12: 235-248.

Corrigan PW and Watson AC (2002) The paradox of self-stigma and mental illness. Clinical Psychology: Science and Practice 9(1): 35-53.

Costea B, Amiridis K and Crump N (2012) Graduate employability and the principle of potentiality: An aspect of the ethics of HRM. Journal of Business Ethics 111(1): 25-36.

Creed WED and Scully MA (2000) Songs of ourselves: Employees' deployment of social identity in workplace encounters. Journal of Management Inquiry 9: 391-413.

Dean M (1999) Governmentality. London: SAGE.

Denissen AM (2010) The right tools for the job: Constructing gender meanings and identities in the male-dominated building trades. Human Relations 67(3): 1051-1069.

Dimoff JK, Kelloway EK and Burnstein MD (2016) Mental Health Awareness Training (MHAT): The development and evaluation of an intervention for workplace leaders. International Journal of Stress Management 23: 167-189.

Eisenhardt KM (1989) Building theories from case study research. Academy of Management Review 14(4): 532-550. 
Elraz H (2016) Studying sensitive issues on mental health at work: The researcher lens. In: Prasad A (ed.) Contesting Intellectual Hegemony in Today' Business Schools. Bingley: Emerald Group Publishing.

Ely RJ and Thomas DA (2001) Cultural diversity at work: The effects of diversity perspectives on work group processes and outcomes. Administrative Science Quarterly 46(2): 229-273.

Equality Act (2010) Definition of Disability under the Equality Act 2010. Available at: https://www.gov.uk/definition-of-disability-under-equality-act-2010 (accessed 24 July 2017).

Evans-Lacko S, Henderson C and Thornicroft G (2013) Public knowledge, attitudes and behaviour regarding people with mental illness in England 2009-2012. The British Journal of Psychiatry 202(55): 51-57.

Forgeard MJ, Pearl RL, Cheung J, et al. (2016) Positive beliefs about mental illness: Associations with sex, age, diagnosis, and clinical outcomes. Journal of Affective Disorders 204: 197-204.

Foster D (2007) Legal obligation or personal lottery? Employee experiences of disability and the negotiation of adjustments in the public sector workplace. Work Employment and Society 21(1): 6784.

Foster DJ and Wass VJ (2013) Disability in the labour market: An exploration of concepts of the ideal worker and organisational fit that disadvantage employees with impairments. Sociology 47(4): 705721.

Foucault M (1977) Discipline and Punish. Sheridan A (trans.). London: Allen Lane.

Foucault M (1982) The subject and power. In: Dreyfus HL and Rabinow P (eds) Michel Foucault Beyond Structuralism and Hermeneutics. New York: Harvester Wheatsheaf, 208-226.

Friskopp A and Silverstein S (1995) Straight Jobs, Gay Lives: Gay and Lesbian Professionals, the Harvard Business School, and the American Workplace. New York: Scribner.

Fryers T, Melzer D, Jenkins R and Brugha T (2005) The distribution of the common mental disorders: Social inequalities in Europe. Clinical Practice \& Epidemiology in Mental Health 1: 14.

Garnsey E and Rees B (1996) Discourse and enactment: Gender inequality in text and context. Human Relations 49(8): 1041-1064.

Gelb BD and Corrigan PW (2008) How managers can reduce mental illness costs by reducing stigma. Business Horizons 51: 293-300.

Ghauri PN and Grønhaug K (2002) Research Methods in Business Studies: A Practical Guide (2 ${ }^{\text {nd }}$ edn). Harlow: Pearson Education Limited.

Goffman E (1968) Stigma: Notes on the Management of Spoiled Identity. Harmondsworth: Pelican Books.

Goldman L and Lewis J (2008) The invisible illness. Occupational Health 60(6): 20-21.

Greene K (2000) Disclosure of chronic illness varies by topic and target: The role of stigma and boundaries in willingness to disclose. In: Petronio S (ed.) Balancing the Secrets of Private Disclosures. Mahwah, NJ: Lawrence Erlbaum Associates, 123-135.

Hardy C and Thomas R (2013) Strategy, discourse and practice: The intensification of power. Journal of Management Studies 51(2): 320-348. 
Hatchard K (2008) Disclosure of mental health. Work 30(3): 311-316.

Holley LC, Stromwall LK and Bashor KH (2012) Reconceptualizing stigma: Toward a critical antioppression paradigm. Stigma Research and Action 2(2): 51-61.

Holmer-Nadesan M (1996) Organizational identity and space of action. Organization Studies 17(1): 49-81.

Honey A (2003) The impact of mental illness on employment: Consumer's perspectives. Work 20(3): 267-276.

Hyde P and Davies H (2004) Service design, culture and performance: Collusion and co-production in health care. Human Relations 57(11): 1407-1426.

Jammaers E, Zanoni P and Hardonk S (2016) Constructing positive identities in ableist workplaces: Disabled employees' discursive practices engaging with the discourse of lower productivity. Human Relations 69(6): 1365-1386.

Janssens M and Zanoni P (2005) 'Many diversities for many service': Theorizing diversity (management) in service companies. Human Relations 58(3): 311-340.

Kessler RC, Chiu WT, Demler O, et al. (2005) Prevalence, severity and comorbidity of 12-month DSMIV disorders in the National Comorbidity Survey Replication. Archives of General Psychiatry 62(6): 617627.

Keyes CLM (2005) Mental illness and/or mental health? Investigating axioms of the complete state model of health. Journal of Consulting and Clinical Psychology 73(3): 539-548.

Kitchener BA and Jorm AF (2004) Mental health first aid training in a workplace setting: A randomized controlled trial. [ISRCTN13249129] BMC Psychiatry 4: 1-8.

Kitchener BA and Jorm AF (2008) Mental Health First Aid: An international programme for early intervention. Early Intervention in Psychiatry 2(1): 55-61.

Knights D and Clarke C (2014) It's a bittersweet symphony, this life: Fragile academic selves and insecure identities at work. Organization Studies 35(3): 335-357.

Knights D and Willmott H (1999) Management Lives: Power and Identity in Contemporary Organizations. London: SAGE.

Kondo DK (1990) Crafting Selves: Power, Gender, Discourses of Identity in a Japanese Workplace. Chicago: Chicago University Press.

Krupa T, Kirsh B, Cockburn L and Gewurtz R (2009) A model of stigma of mental illness in employment. Work 33(4): 413-425.

Leufstadius C, Eklund M and Erlandsson LK (2009) Meaningfulness in work - experiences among individuals with persistent mental illness. Work 34(1): 21-32.

Lloyd C (ed.) (2010) Vocational Rehabilitation and Mental Health. Oxford: Wiley Blackwell.

Marmot Review (2010) Global Commission on Social Determinants of Health. Post-2010. Available at: http://webarchive.nationalarchives.gov.uk/+/http://www.dh.gov.uk/en/Publichealth/Healthinequali ties/DH_094770 (accessed 24 July 2017). 
Martin A, Woods M and Dawkins S (2015) Managing employees with mental health issues: Identification of conceptual and procedural knowledge for development within management education curricula. Academy of Management Learning and Education 14(1): 50-68.

Matthews CK and Harrington NG (2000) Invisible disability. In: Braithwaite DO and Thompson TL (eds) Handbook of Communication and People with Disabilities. Mahwah, NJ: Lawrence Erlbaum Associates, 405-421.

Mausner-Dorsch H and Eaton WW (2000) Psychosocial work environment and depression: Epidemiologic assessment of the demand-control model. American Journal of Public Health 90(11): $1765-1770$.

Mindful Employer (2016) Making work work. Available at: http://www.mindfulemployer.net/files/4014/2692/7677/Making_work_work.pdf (accessed 24 July 2017).

Munro I (2012) The management of circulations: Biopolitical variations after Foucault. International Journal of Management Reviews 14(3): 345-362.

Musson G and Duberley J (2007) Change, change or be exchanged: The discourse of participation and the manufacture of identity. Journal of Management Studies 44(1):143-165.

NIMH (2015) National Institute for Mental Health strategic plan for research. NIH Publication

Number 15-6368. Available at: https://infocenter.nimh.nih.gov/nimh/product/Nationallnstitute-ofMental-Health-Strategic-Plan-For-Research/NIH\%2015-6368 (accessed 24 July 2017).

Occupational Health (2006) Workers fear revealing mental illness issues. Occupational Health 58(11): 7.

Omansky-Gordon B and Rosenblum KE (2001) Bringing disability into the sociological frame: A comparison of disability with race, sex, and sexual orientation statuses. Disability \& Society 16(1): 519.

Overboe J (1999) 'Difference in itself': Validating the lived experience of disabled people. Body and Society 5(4): 17-29.

Pinfold V, Thornicroft G, Huxley $\mathrm{P}$ and Farmer $\mathrm{P}$ (2005) Active ingredients in anti-stigma in programmes in mental health. International Review of Psychiatry 17(2): 123-131.

Randall J and Munro I (2010) Foucault's care of the self: A case from mental health work. Organization Studies 31(11): 1485-1504.

Riach K (2007) 'Othering' older worker identity in recruitment. Human Relations 60(11): 1701-1726.

Roberts M (2005) Time, human being and mental health care: An introduction to Gilles Deleuze. Nursing Philosophy 6(3): 161-173.

Rose N (1999) Governing the Soul: The Shaping of the Private Self (2nd edn). London: Routledge.

Rumens N and Kerfoot D (2009) Gay men at work: (Re)constructing the self as professional. Human Relations 62(5): 763-786.

Santuzzi AM and Waltz PR (2016) Disability in the workplace: A unique and variable identity. Journal of Management 42(5): 1111-1135. 
Scull AT (1979) Museums of Madness: The Social Organization of Insanity in Nineteenth Century England. London: Allen Lane.

Slay HS and Smith DA (2011) Professional identity construction: Using narrative to understand the negotiation of professional and stigmatized cultural identities. Human Relations 64(1): 85-107.

Starkey K and Hatchuel A (2002) The long detour: Foucault's history of desire and pleasure. Organization 9(4): 641-656.

Taylor V and Raeburn NC (1995) Identity politics as high-risk activism: Career consequences for lesbian, gay, and bisexual sociologists. Social Problems 42(2): 252-273.

The Lawyer (2009) The mental health stigma in the workplace. Available at: http://www.thelawyer.com/opinion-the-mental-healthstigma-intheworkplace/1001666.article (accessed 24 July

2017).

The Sainsbury Centre for Mental Health (2007) Mental Health at Work. Available at: http://www. impact.ie/wp-content/uploads/2015/07/Mental-Health-at-Work.pdf (accessed 24 July 2017).

Thoits PA (2016) 'I'm not mentally ill': Identity deflection as a form of stigma resistance. Journal of Health and Social Behavior 57(2): 135-151.

Thomas R (2009) Critical studies on identities: Mapping the terrain. In: Alvesson M, Bridgman T and Willmott $\mathrm{H}$ (eds) The Oxford Handbook of Critical Management Studies. Oxford: Oxford University Press, 166-185.

Thomas R and Davies A (2005) Theorizing the micro-politics of resistance: Discourses of change and professional identities in the UK public services. Organization Studies 26(5): 683-706.

Time to Change (2014) National attitudes to mental illness. Available at: https://www.timetochange.org.uk/news/survey-shows-greatest-improvement-public-attitudes-mental-healthdecade (accessed 24 July 2017).

Trudel L, Vonarx N, Simard C, et al. (2009) The adverse effects of psychosocial constraints at work: A participatory study to orient prevention to mitigate psychological distress. Work 34(3): 345-357.

Tsang HWH, Angell B, Corrigan P, et al. (2007) A cross-cultural study of employers' concerns about hiring people with psychotic disorder: Implication for recovery. Social Psychiatry and Psychiatric Epidemiology 42(9): 723-733.

Tse S (2004) What do employers think about employing people with experience of mental illness in New Zealand workplaces? Work 23(3): 267-274.

Van Laer K and Janssens M (2011) Ethnic minority professionals' experiences with subtle discrimination in the workplace. Human Relations 64(9): 1203-1227.

Zanoni P and Janssens M (2004) Deconstructing difference: The rhetoric of human resource managers' diversity discourses. Organization Studies 25(1): 55-74. 